\section{Spreading the tau}

Filamentous inclusions composed of phosphorylated tau are present in several neurodegenerative diseases. In the brains of patients with Alzheimer's disease, these inclusions seem to 'spread' from one region to another in a characteristic pattern as the disease progresses. Goedert, Tolnay and colleagues now provide the first in vivo evidence that tau aggregation can spread between connected cells through the transmission of tau 'seeds'.

The authors injected brain extracts from mice expressing P301S tau, a mutated form of tau that causes inherited frontotemporal dementia, into the brains of ALZ17 mice, which

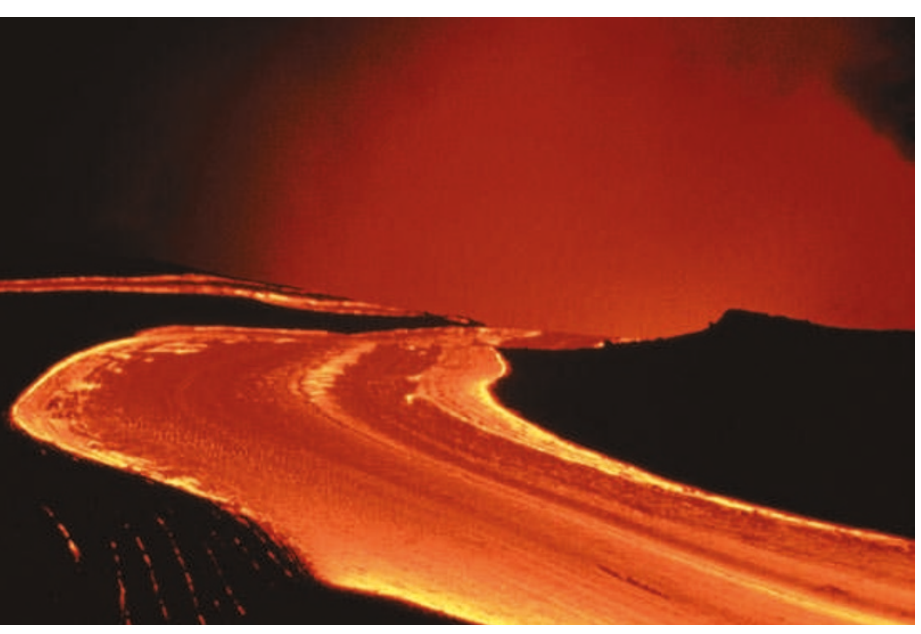

express a form of human tau that does not normally aggregate. Over a period of 6-15 months after the injection, increasing numbers of filamentous hyperphosphorylated tau deposits formed in the brains of these mice, whereas those of control mice (which received brain extracts from P301S mice from which the mutant tau had been depleted) were free of inclusions. Tau aggregation was induced both at the injection site in the hippocampus or cortex and in anatomically connected regions up to $4 \mathrm{~mm}$ from the injection site, including the fimbria, the optic tract, the thalamus, the cerebral peduncle and the hypothalamus.

The deposits were composed of the tau isoform that is normally expressed by ALZ17 mice, indicating that the injected brain extract induced the aggregation of wild-type tau. The authors also showed that insoluble forms of P301S tau had a much greater capacity to induce tau aggregation than soluble forms of the protein.

Interestingly, the injected mice showed no overt signs of neurodegeneration, such as neuron loss or axon damage, within the timeframe studied. The authors suggest that this may be because the "tau species responsible for transmission and neurotoxicity are not identical". Future work, such as electrophysiological studies, might determine whether functional defects that might lead to later neurodegeneration are present in the affected regions.

This study confirms that, as previously demonstrated in vitro, tau aggregation can be transmitted from cell to cell in vivo. Similar mechanisms have been proposed to contribute to the distribution of $\alpha$-synuclein inclusions in Parkinson's disease, and so targeting this transmission might be a viable therapeutic strategy for both of these diseases. Several issues will, however, need to be addressed, including the nature of the transmissible tau seeds, how they pass from one cell to another and whether distinct strains of tau (like different strains of prion protein) have different capacities to induce aggregation and cause neurodegeneration. In addition, the model of tau transmission presented in this paper may be used in future work to determine the relationship between tau aggregation and disease.

\section{Katherine Whalley}

ORIGINAL RESEARCH PAPER Clavaguera, F. et al. Transmission and spreading of tauopathy in transgenic mouse brain. Nature Cell Biol.11, 909-913 (2009) 